\section{Successful management of a foreseeable and preventable complication of transcatheter valve implantation}

\author{
Ali Rıza Akyüz (D), Levent Korkmaz (D) \\ Department of Cardiology, University of Health Sciences, Trabzon Ahi Evren \\ Cardiovascular and Thoracic Surgery Training and Research Hospital; \\ Trabzon-Turkey
}

\section{Introduction}

The Myval THV is a new balloon expandable valve which has obtained the European Community (CE) mark following the Myval-1 study (1). In this study, 30 patients with severe aortic stenosis (AS) in the medium to high risk group were treated by Myval without major complications such as mortality, paravalvular leakage (PVL), or the need for permanent pacemakers (PPM). These data are similar to the percutaneous arm of the PARTNER-3 trial (2).

At the beginning of the transcatheter aortic valve implantation (TAVI) procedure, predilatation was commonly recommended and applied before the implantation of valve. After gaining experience and improvement in valve designs, many operators prefer not to predilate and implant the valve directly; however, there are still no well-defined criteria regarding which valve should be predilatated. We generally consider the degree of calcification, valve area, and mean gradient as important factors to decide whether predilatation is necessary or not. In addition, many studies and personal experiences approve direct TAVI to be as effective and safe as routine TAVI procedures with predilatation (3).

\section{Case Report}

An 80-year-old male patient was admitted to our hospital with severe shortness of breath and pulmonary edema. The patient's past medical history included type 2 diabetes mellitus, hypertension, permanent pacemaker implantation, and dyslipidemia. Echocardiographic examination revealed a maximum gradient of $68 \mathrm{~mm} \mathrm{Hg}$ and an average of $40 \mathrm{~mm} \mathrm{Hg}$, and the aortic valve area was calculated as $0.8 \mathrm{~cm}^{2}$. The decision of the heart team was to treat this patient with TAVI.

Based on tomographic assessment, the valve was not severely calcified even though mean gradient was high, and the 26-mm Myval was chosen for treatment according to measurement of the area. Right femoral artery was chosen for operation and 26-mm Myval implantation was planned without predilatation. During advancing the valve through sheath, we faced significant friction and noticed that the valve moved back and de- centralized slightly over the balloon (Fig. 1a). We sent the valve to the aortic root, but the prosthesis valve could not cross the aortic valve despite many attempts. As we pulled the unimplanted valve back through the sheath gently, it was dislodged from the balloon (Video 1). After a careful evaluation, we noticed that the prosthesis did not enter the sheath because distal part of the sheath was not clearly seen on x-ray and blocked the valve entrance and removed the valve from the balloon even though we did not apply huge pressure.

We tried to send the balloon through the valve but failed (Video 2). Hence, a long sheath was placed in the right brachial artery, and a hydrophilic wire was sent through the valve delivery shaft. The snare was advanced through the long sheath, and the wire was captured (Fig. 1b). We sent a $7 \times 100 \mathrm{~mm}$ size peripheral balloon through the long sheath to dilate the valve at least partially (Fig. 1c) to allow the original balloon to cross the valve and implant it. After dilatation with the peripheral balloon, we could only partially cross the valve with the original balloon and dilate the valve. Further dilatation could only be done with CODA balloon catheter (Cook Incorporated, Bloomington, IN, United States) (Fig. 1d) after removing the delivery and Myval balloon. The second valve was then implanted successfully after predilation (Video 3 ).

\section{Discussion}

Operators generally tend to avoid predilation of native aortic valve because of potential complications and with the aim of de-

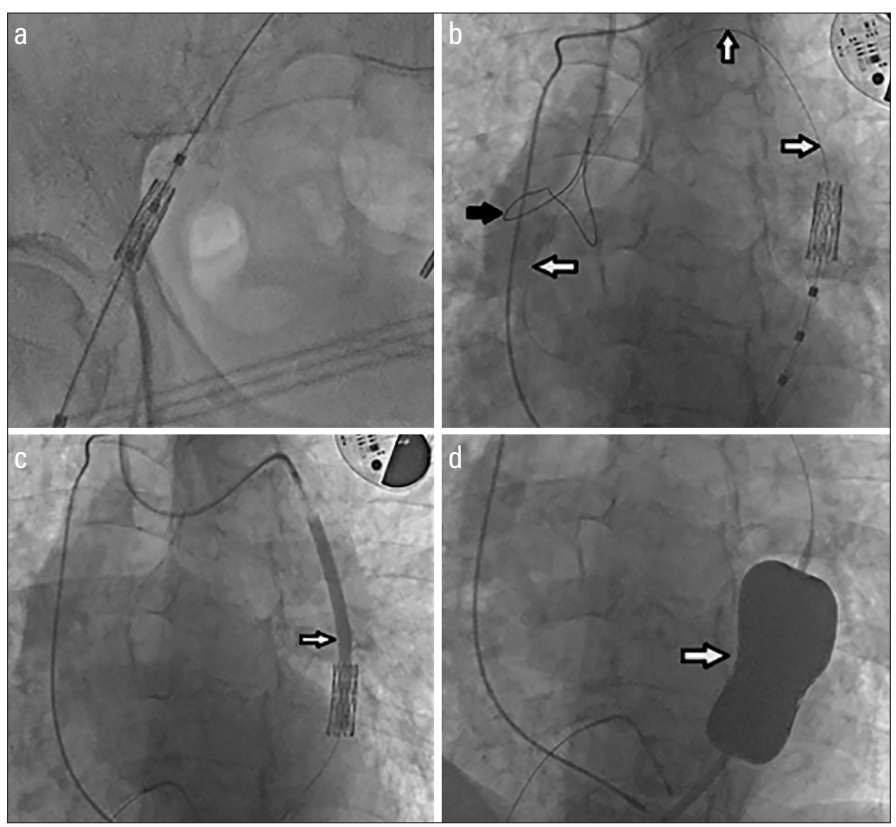

Figure 1. (a) The valve slipped backwards from the balloon. (b) Capturing the hydrophilic wire (white arrows) sent from the valve shaft with a macro snare (black arrow). (c) Partial dilation of the prosthesis with a $7 \times 100 \mathrm{~mm}$ peripheral balloon (arrow). (d) Implantation of the valve to the descending aorta with a Coda balloon (arrow) 
creasing the length of procedure. However, significant problems may arise if the prosthesis valve cannot cross the aortic valve, especially if retrieval valves are used. In such situations, a feasible solution is to predilate the valve using different arteries, rather than the one where TAVI valve is placed. In other situations, like in our patient, getting the valve back inside the sheath and removal of the valve with the sheath would be another option (4).

The main reason why we did not predilate the valve was the lack of severe calcification. Furthermore, taking our previous experiences into account, Myval could cross easily and we rarely used predilatation until that time. In addition, we pulled the valves back in two different patients who were heavily calcified and not predilated. In one patient, the valve came back easily without removing the sheath from the artery; however, in the other patient, we had to remove the sheath because valve got stuck inside the sheath.

There could be a number of reasons why this complication occurred. First, Myval is mounted over the balloon outside the patient. When introducing the mounted valve through the sheath, there is a possibility of valve movement. In our patient, we saw it but paid no attention. That complication could have been averted if we had not advanced the valve when we saw the movement of the valve inside the sheath. In such situations, our current approach is to pull the valve back and mount it again. We also routinely predilate the sheath with $18 \mathrm{~F}$ dilatation before introducing the valve, and it results in significant decrease in friction; and no backward movement of valve has been detected till this time. Second, predilatation should be carefully considered, especially if non-retrievable valves are used. Finally, the distal part of the sheath should be more recognizable.

\section{Conclusion}

We believe that the main reason for this complication could be attributable to the unintentional movement of the valve during its advancement in the sheath. It can be avoided by routinely predilatating the sheath before introducing the valve.
Informed consent: Written informed consent to publication was obtained from the patient.

Video 1. Stripping of the valve from the balloon by attaching the sheath to its distal end during valve retrieval

Video 2. Trying to load the balloon by resting the prosthetic cover on the native cover

Video 3. Aortographic appearance of both valves after successful implantation

\section{References}

1. Sharma SK, Rao RS, Chandra P, Goel PK, Bharadwaj P, Joseph G, et al.; Collaborators. First-in-human evaluation of a novel balloonexpandable transcatheter heart valve in patients with severe symptomatic native aortic stenosis: the MyVal-1 study. Eurolntervention 2020; 16: 421-9. [Crossref]

2. Mack MJ, Leon MB, Thourani VH, Makkar R, Kodali SK, Russo M, et al.; PARTNER 3 Investigators. Transcatheter Aortic-Valve Replacement with a Balloon-Expandable Valve in Low-Risk Patients. N Engl J Med 2019; 380: 1695-705. [Crossref]

3. Leclercq F, Robert P, Akodad M, Macia JC, Gandet T, Delseny D, et al. Prior Balloon Valvuloplasty Versus Direct Transcatheter Aortic Valve Replacement: Results From the DIRECTAVI Trial. JACC Cardiovasc Interv 2020; 13: 594-602. [Crossref]

4. Sharma SK, Rao RS, Chopra M, Sonawane A, Jose J, Sengottuvelu G. Myval transcatheter heart valve system in the treatment of severe symptomatic aortic stenosis. Future Cardiol 2021; 17: 73-80. [Crossref]

Address for Correspondence: Dr. Ali Rıza Akyüz, Sağlık Bilimleri Üniversitesi, Trabzon Ahi Evren Göğüs Kalp ve Damar Cerrahisi Sağlık Uygulama ve Araştırma Merkezi, Kardiyoloji Kliniği, Trabzon-Türkiye

Phone: +905065051619

E-mail:dralirizaakyuz@gmail.com

(C) Copyright 2021 by Turkish Society of Cardiology -

Available online at www.anatoljcardiol.com

DOI:10.5152/AnatolJCardiol.2021.37383 